\title{
A mathematical model of Doxorubicin treatment efficacy for non-Hodgkin's lymphoma: investigation of the current protocol through theoretical modelling results
}

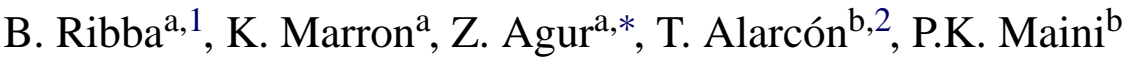 \\ anstitute for Medical BioMathematics, 10 Hate'ena Street, P.O.B. 282, 60991 Bene Ataroth, Israel \\ b Centre for Mathematical Biology, Mathematical Institute, University of Oxford, 24-29 St. Giles', \\ Oxford OX1 3LB, UK
}

Received 31 July 2003; accepted 17 June 2004

\begin{abstract}
Doxorubicin treatment outcomes for non-Hodgkin's lymphomas (NHL) are mathematically modelled and computationally analyzed. The NHL model includes a tumor structure incorporating mature and immature vessels, vascular structural adaptation and NHL cell-cycle kinetics in addition to Doxorubicin pharmacokinetics (PK) and pharmacodynamics (PD). Simulations provide qualitative estimations of the effect of Doxorubicin on high-grade (HG), intermediate-grade (IG) and low-grade (LG) NHL. Simulation results imply that if the interval between successive drug applications is prolonged beyond a certain point, treatment will be inefficient due to effects caused by heterogeneous blood flow in the system.

(c) 2004 Society for Mathematical Biology. Published by Elsevier Ltd. All rights reserved.

\footnotetext{
* Corresponding author.

E-mail address: agur@imbm.org (Z. Agur).

1 Also at: Clinical Pharmacology Unit, Faculty of Medicine Laënnec, University of Lyon, Paradin Street, P.O.B. 8071, 69376 Lyon, France.

2 Also at: Bioinformatics Unit, Department of Computer Science, University College London, Gower Street, London WC1E 6BT, UK.
} 


\section{Introduction}

Non-Hodgkin's lymphomas (NHL) are neoplastic transformations of lymphoid tissue cells (Bast et al., 2000). In 2003, 60,000 cases of NHL were expected to be diagnosed in the United States, where it ranks as the sixth leading cause of cancer-related death (Boring et al., 1994). Less than $35 \%$ of all diagnosed cases are cured; hence the treatment of this disease poses a difficult clinical problem. Currently, NHL patients are treated with so-called CHOP chemotherapy (Cyclophosphamide, Hydroxydoxorubicin, Oncovin, Prednisone) in which Hydroxydoxorubicin (Doxorubicin) and Cyclophosphamide are the more active drugs (Lepage et al., 1993). CHOP is usually administered over a total of 6 to 8 cycles separated by 21 day intervals (Couderc et al., 2000). The relationship between this dosing interval and the efficacy of NHL CHOP treatment has not been systematically analyzed. However, theory suggests that the success of cancer chemotherapy is primarily determined by the frequency of drug administration (Agur, 1985; Agur et al., 1988; Cojocaru and Agur, 1992). For this reason we attempt to assess the efficacy of the CHOP protocol by means of a more systematic approach based on mathematical modelling and computersimulation.

Extensive studies concerning the modelling of vascular tumor growth have previously been carried out by Liotta et al. (1977), Orme and Chaplain (1996) and recently by Arakelyan et al. (2002). In this work, we propose a two-dimensional mathematical model aimed at simulating the effect of Doxorubicin on NHL. The framework that we use is that of the hybrid cellular automata (CA), which has already been applied to different aspects of tumor growth (Anderson and Chaplain, 1998; Anderson et al., 2000; Patel et al., 2001; Deutsch and Dormann, 2002; Alarcón et al., 2003).

In our model, chemicals (nutrient and drug) and flow-related quantities are represented as continuum variables, whereas cells and vessels are considered as individual elements. The model distinguishes between three different grades of NHL: Low Grade (LG), which progresses slowly and is the least aggressive, High Grade (HG), which is very aggressive and progresses over a short period of time, and Intermediate Grade (IG), which is moderately aggressive. Clinical studies have shown differences in the cell-cycle kinetic profiles of the three grades, which are implemented in the model (see Table 1).

We consider a computational domain composed of a vascular network filled with NHL cells. The vascular network supplies the NHL cells with blood-borne nutrients and drugs. The model takes into account two key factors influencing the efficiency of drug delivery. The first is the coupling of NHL growth with the vascular network (Yancopoulos et al., 2000), which affects the configuration of the blood vessels, i.e., the creation of mature or immature vessels. The second is the blood flow heterogeneity which results from this diverse construction (Jain, 2001; McDougall et al., 2002).

The dynamics of the NHL cell colony are determined by the cell-cycle kinetics and by the nutrient and drug concentrations available through diffusion from the vasculature. Doxorubicin pharmacokinetics (PK) were investigated in order to accurately determine the drug concentration in the vasculature at any given time. Doxorubicin pharmacodynamics (PD) were investigated as well, in order to simulate the qualitative effect of the drug on NHL cells. 

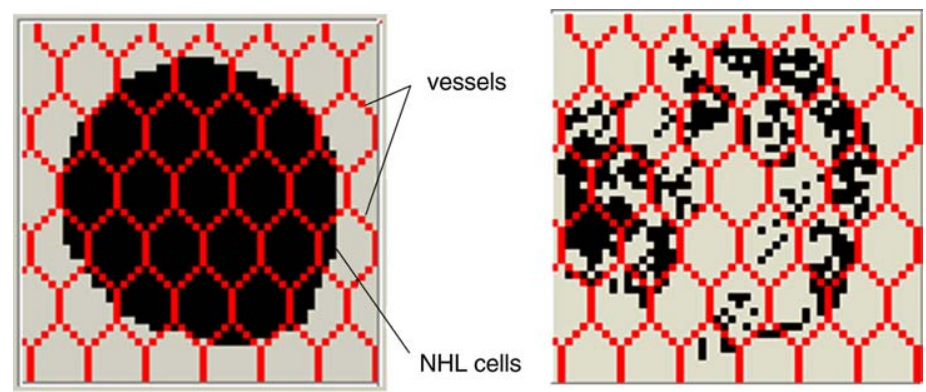

Fig. 1. Representation of NHL cells and the vascular network on the computational domain. Black areas represent cancer cells, grey lines (red lines in the web version) represent blood vessels. Left: a fully populated domain; right: the domain following significant cell depletion.

\section{Mathematical model description}

We consider a $2 \mathrm{~mm}$ square tissue (2D model) composed of 110 vessels, producing a density in accordance with quantitative estimations. Vessels compose a simple vascular network, as has been observed in vivo (Honda and Yoshizato, 1997). A similar 'honeycomb' vascular structure has been observed in liver and colon tissues (Konerding et al., 2001). Hence, it is reasonable to assume that the basic vascular structure will not change significantly throughout the initial stages of tumor development.

The vessels' radii are set initially at $20 \mu \mathrm{m}$ (Willemse et al., in press) and are subject to modification through vessel structural modification processes. The domain is initially filled with NHL cells forming unified core structures or more scattered patterns (see Fig. 1).

\subsection{Vessel structural modification}

In our model the vasculature is subject to remodelling, which occurs according to two different mechanisms. The first is the so-called structural adaptation mechanism, studied by Pries et al. (1994, 1998) and then by Alarcón et al. (in preparation).

Normal vasculature is endowed with smooth muscle cells and pericytes, which allow blood vessels to contract and expand. This adaptation is assumed to occur in response to three stimuli (Pries et al., 1994): hemodynamic, metabolic and a 'shrinking tendency'. The hemodynamic stimulus corresponds to the tendency of the vascular system to maintain a constant wall shear-transmural pressure relationship. The metabolic stimulus corresponds to the response of the vessel to tissue demands, and the 'shrinking tendency' signifies vessel shrinkage which occurs in the absence of growth factors.

The second vascular remodelling mechanism that we implement is vessel maturation and the destabilization of mature vessels. In cancer, due to neovascularization, a significant proportion of the vessels are immature (Benjamin et al., 1999). Unlike mature vessels, immature vessels are not encased in pericytes and smooth muscle cells. Therefore we consider them as incapable of structural adaptation. As a result, their configuration is less stable: their radii are subject to random change and tend to be larger than the radii of mature vessels, increasing leakage. In order to incorporate these characteristics, the 
immature vessels' radii are modified at each time step of the simulation according to the following process:

$$
r_{i m}=r_{\text {mat }} \cdot(1+\epsilon)
$$

where $r_{i m}$ stands for the immature vessel radius and $r_{\text {mat }}$ is the initial radius of the mature vessels, taken to be $20 \mu \mathrm{m} . \epsilon$ is a random number uniformly distributed in the interval $(0,3)$ according to Willemse et al. (in press). As the NHL cell colony grows it engulfs nearby blood vessels. Since cancer cells have the ability to destabilize mature vessels (Yancopoulos et al., 2000), we have considered that once a vessel is surrounded by NHL cells it immediately becomes immature. The status of a vessel (mature or immature) is updated at each time step of simulation, thus allowing immature vessels to become mature once again, as occurs in actual vascular tumors (Yancopoulos et al., 2000).

\subsection{Blood flow}

We assume the flow in each vessel to be laminar steady Poiseuille flow, i.e.,

$$
\begin{aligned}
& \Delta P=\dot{Q} Z \\
& Z=\frac{8 \mu(r, H) L}{\pi r^{4}}
\end{aligned}
$$

where $\Delta P$ is the pressure drop between two points of the network, $\dot{Q}$ the flow rate in each vessel, $Z, L, r$ and $H$ are respectively the resistance, length, radius and hematocrit. ${ }^{3} \mu$ is the radius and hematocrit dependent viscosity (Alarcón et al., 2003).

\subsection{Diffusion of nutrients and drug}

We make the biologically realistic assumption that nutrient and drug molecules reach their equilibrium state on a timescale far shorter than that of the cell dynamics. This assumption allows us to apply the so-called adiabatic approximation, according to which these chemicals can be considered instantaneously in the steady state. Hence, the diffusion of drug and nutrient molecules has been described by successive solutions of an elliptic boundary value problem.

Considering a two-dimensional domain, let $C(x, y, t)$ be the concentration of nutrient or drug. According to the adiabatic approximation, the corresponding diffusion equation can be written as follows:

$$
D_{p} \nabla^{2} C(x, y, t)-k(x, y) \cdot C(x, y, t)=0
$$

where $D_{p}$ is the diffusion coefficient and $k(x, y)$ the uptake coefficient at position $(x, y)$.

We prescribe appropriate boundary conditions for Eq. (4) assuming that nutrient and drug molecules enter the system by crossing the vessel walls, the flux being given by

$$
\vec{J}=-D_{p} \nabla C .
$$

\footnotetext{
3 The hematocrit is defined as the fraction of the volume of blood occupied by red blood cells.
} 
Thus, we impose the following mixed boundary conditions on the vessel walls:

$$
-D_{p} \mathbf{n}_{\mathbf{w}} \cdot \nabla C(x, y, t)=P \cdot\left(C_{b}-C\right)
$$

where $\mathbf{n}_{\mathbf{w}}$ is the unit outward vector, orthogonal to the vessel wall, $P$ is the permeability of the vessel, assumed to be the same for both nutrient and drug, and $C_{b}$ is the drug or nutrient concentration in the plasma.

We also impose no-flux boundary conditions along the edges of our computational domain, $\Omega$ :

$$
\left.\mathbf{n}\right|_{\partial \Omega} \cdot \nabla C(x, y, t)=0
$$

where $\left.\mathbf{n}\right|_{\partial \Omega}$ is the unit outward vector, orthogonal to the boundary of the domain.

This diffusion equation was solved by means of a so-called two-grid V-cycle multigrid method. The multigrid method enables improvement of the rate of convergence of classical numerical methods through interpolation of an initially 'rough' solution on a fine grid (Hackbusch, 1985; McCormick, 1987).

\subsection{Doxorubicin pharmacokinetics $(P K)$}

We use the so-called one-compartment model in which the decline of drug concentration in plasma over time is described by

$$
\begin{aligned}
& \frac{\partial C_{b}}{\partial t}=-k \cdot C_{b}(t) \\
& C_{b}(0)=\frac{\text { dose }}{V_{d}}
\end{aligned}
$$

where $V_{d}$ is the volume of distribution of the drug and $k$ the fraction of drug removed from the compartment per unit time, inversely related to the half-life $t_{1 / 2}$ (Hardman et al., 2001).

\subsection{Doxorubicin pharmacodynamics $(P D)$}

Doxorubicin is known to act on both proliferative and quiescent cells (Barranco, 1984); however, the effect on quiescent cells can be assumed negligible. The dependence of the survival fraction $(s)$, or the percentage of cells that survives the drug, on drug concentration has been modelled (Hardman et al., 2001) by

$$
s=a+\frac{1}{b+k_{d} \cdot C_{b}}
$$

where $a, b$ and $k_{d}$ are positive constants set according to Kwok and Twentyman (1985) and Nagai et al. (2002).

Because the model considers cells as individuals, the survival fraction was interpreted as the probability for one cell to survive the treatment.

\subsection{Cell population dynamics}

We assume that the initial cell colony is composed of NHL cells which can be divided into two categories:

- proliferative cells that progress through the cell cycle;

- quiescent cells. 
Table 1

NHL classification, clinical values and biological ranges (Brons et al., 1992)

\begin{tabular}{llllll}
\hline & S-DNA $(\%)$ & GF $(\%)$ & $T_{S}(\mathrm{~h})$ & $T_{c}(\mathrm{~h})$ & $T_{G_{1}}(\mathrm{~h})$ \\
\hline HG NHL & 25.2 & 34 & 19.1 & 57 & 36 \\
& $(3.6-37.1)$ & $(15-93)$ & $(9.7-33.6)$ & $(21-93)$ & $(11-59)$ \\
IG NHL & 10.2 & 26 & 13.4 & 69 & 47 \\
& $(1.9-35.2)$ & $(11-85)$ & $(8.6-50.2)$ & $(20-270)$ & $(13-215)$ \\
LG NHL & 1.2 & 6 & 12.8 & 107 & 80 \\
& $(0.4-4.2)$ & $(3-36)$ & $(8.4-16.5)$ & $(22-122)$ & $(16-93)$ \\
\hline
\end{tabular}

The initial growth fraction (GF), the initial percentage of S-phase (S-DNA) and the cycle-phase duration ( $T_{S}$ for S-phase, $T_{G_{1}}$ for G1-phase, $T_{G_{2}}$ for G2-phase, $T_{M}$ for Mphase and $T_{c}$ for total cell cycle) were set according to various NHL cell-cycle kinetic studies (Brons et al., 1992; Erlanson et al., 1995; Stokke et al., 1998) (see Table 1).

The progression of each proliferative cell through its cycle is modelled by the following CA rules:

- The probability of cell death is determined by the drug's concentration and PD.

- If the cell is not killed by the drug, it advances one time step in its cycle phase.

- Between G1 and the S-phase [R point restriction (Blagosklonny and Pardee, 2002)], the cell can either die, be arrested or continue progressing through the cell cycle according to its local environment (Bast et al., 2000), i.e., local nutrient concentration and overcrowdedness (Alberts et al., 1994).

- If the environmental conditions are appropriate, the cell enters G2 and divides, daughter cells moving towards higher nutrient concentrations.

In order to reduce computational complexity, we assume that only glucose regulates cell proliferation and death, and do not consider the effect of oxygen distribution. This is also due to the apparent unpredictability of red blood cell concentration downstream from microvascular bifurcations (Enden and Popel, 1994).

\subsection{Cell-cycle kinetic profiles}

According to our model assumptions, the cell-cycle kinetics which determine the growth of the cell colony are regulated by the nutrient flow available to the cell (see above). If nutrient flow decreases below a certain level, the cell will become quiescent and may even die. Our model simulates this effect by means of a 'nutrient block parameter' $\left(n_{\text {block }}\right)$ and a 'nutrient death parameter' $\left(n_{\text {death }}\right)$. Each grade of NHL was attributed its own $n_{\text {block }}$ and $n_{\text {death }}$ values (see Table 2). Due to a lack of information on the regulation of cell death at a macroscopic level, we estimated a constant value for $n_{\text {death }}$. The value of $n_{\text {block }}$ was then derived as follows: several simulations of NHL behavior with no Doxorubicin effect were run per grade, each one testing a different $n_{\text {block }}$ value. After each simulation, we computed the average growth fraction (GF) and the average percentage of cells in the $S$ cell-cycle phase (S-DNA). The $n_{\text {block }}$ value which produced results most similar to those obtained in clinical studies was selected (see Table 1). 
Table 2

Cell-cycle kinetic profiles for simulated NHL cell dynamics in a drug-free domain (Brons et al., 1992)

\begin{tabular}{|c|c|c|c|c|}
\hline & $n_{\text {block }}\left(\mathrm{mM} \mathrm{s}^{-1}\right)$ & $n_{\text {death }}\left(\mathrm{mM} \mathrm{s}^{-1}\right)$ & S-DNA (\%) & $\mathrm{GF}(\%)$ \\
\hline HG NHL & 0.0066 & 0.001 & 11.9 & 39.2 \\
\hline IG NHL & 0.0066 & 0.001 & 3.5 & 20.6 \\
\hline LG NHL & 0.009 & 0.001 & 0.7 & 7.9 \\
\hline
\end{tabular}

Table 3

Model parameters

\begin{tabular}{|c|c|c|c|c|c|}
\hline Equation & Parameter & Description & Unit & Value & Reference \\
\hline (1) & $r_{\text {mat }}$ & $\begin{array}{l}\text { Mature vessel } \\
\text { radius }\end{array}$ & $\mu \mathrm{m}$ & 20 & Willemse et al. (in press) \\
\hline (1) & $r_{i m}$ & $\begin{array}{l}\text { Immature vessel } \\
\text { radius }\end{array}$ & $\mu \mathrm{m}$ & $20 \leq r_{\mathrm{im}} \leq 60$ & Willemse et al. (in press) \\
\hline (6) & $C_{b}$ & $\begin{array}{l}\text { Glucose concen- } \\
\text { tration in blood }\end{array}$ & $\mathrm{mM}$ & 5 & Wilson and Foster (1992) \\
\hline (6) & $P$ & $\begin{array}{l}\text { Vessel perme- } \\
\text { ability }\end{array}$ & $\mathrm{cm} \mathrm{s}^{-1}$ & $3.0 \times 10^{-4}$ & Crone and Levitt (1984) \\
\hline (9) & $V_{d}$ & $\begin{array}{l}\text { Doxorubicin } \\
\text { volume of } \\
\text { distribution }\end{array}$ & $\mathrm{L} \mathrm{m}^{-2}$ & 682 & Hardman et al. (2001) \\
\hline (9) & $t_{1 / 2}$ & $\begin{array}{l}\text { Doxorubicin } \\
\text { half-life }\end{array}$ & $\mathrm{h}$ & 26 & Hardman et al. (2001) \\
\hline (4) & $D_{p}$ & $\begin{array}{l}\text { Glucose } \\
\text { diffusion } \\
\text { coefficient }\end{array}$ & $\mathrm{cm}^{2} \mathrm{~s}^{-1}$ & $2.3 \times 10^{-7}$ & Casciari et al. (1998) \\
\hline (4) & $k$ & $\begin{array}{l}\text { Glucose uptake } \\
\text { coefficient }\end{array}$ & $\mathrm{mM} \mathrm{min}^{-1}$ & $13.2 \times 10^{-11}$ & Lapela et al. (1995) \\
\hline (4) & $D_{p}$ & $\begin{array}{l}\text { Doxorubicin } \\
\text { diffusion coeffi- } \\
\text { cient }\end{array}$ & $\mathrm{cm}^{2} \mathrm{~s}^{-1}$ & $2.7 \times 10^{-10}$ & Lankelma et al. (2000) \\
\hline (10) & $a$ & $\begin{array}{l}\text { Doxorubicin PD } \\
\text { coefficient }\end{array}$ & . & $5 \times 10^{-3}$ & Kwok and Twentyman (1985) \\
\hline (10) & $b$ & $\begin{array}{l}\text { Doxorubicin PD } \\
\text { coefficient }\end{array}$ & $\mathrm{L} \mathrm{mg}^{-1}$ & 1.005 & Kwok and Twentyman (1985) \\
\hline \multirow[t]{2}{*}{ (10) } & $k_{d}$ & $\begin{array}{l}\text { Doxorubicin PD } \\
\text { coefficient }\end{array}$ & $\mathrm{L} \mathrm{mg}^{-1}$ & 2.603 & Kwok and Twentyman (1985) \\
\hline & & $\begin{array}{l}\text { Doxorubicin } \\
\text { effective } \\
\text { concentration }\end{array}$ & $\mathrm{mg} \mathrm{L}^{-1}$ & 0.39 & Nagai et al. (2002) \\
\hline
\end{tabular}

\subsection{Additional parameter values}

Table 3 contains additional parameter values implemented in the model, all of which have been taken from clinical references. The influence of certain critical parameter values on the model's behavior, including estimated parameter values such as $n_{\text {block }}$ and $n_{\text {death }}$ (see above), will be presented in Section 4. 


\section{Evaluation of treatment efficacy}

Due to inevitable model simplification, we could not validate our simulation results by comparing them to those obtained in clinical studies. Nevertheless, we attempted to qualitatively evaluate treatment efficacy for cell colonies by analyzing four kinds of results. Overall treatment effect: We define the overall treatment effect on NHL as

$$
\text { treatment effect }=\frac{n^{\prime}-n}{n} \times 100
$$

where $n$ and $n^{\prime}$ are, respectively, the number of cells at the beginning and at the end of the simulation. This formulation indicates the relative response of NHL colonies to treatment. NHL cell regrowth following Doxorubicin application: After each drug application, we calculated the NHL cell population's regrowth rate, i.e., the increase in the number of cells which occurred once the effect of the drug had worn off. This regrowth was evaluated by computing the difference between the minimum number of cells following drug administration (maximum drug effect) and the number of cells immediately before the next drug cycle, that is,

$$
\left.R_{1}\right|_{(x)}=\frac{\left.n\right|_{(x+1)}-\left.n^{\prime}\right|_{(x)}}{\left.n^{\prime}\right|_{(x)}} \times 100
$$

where $\left.n\right|_{(x+1)}$ is the number of cells just before drug cycle $(x+1)$ and $\left.n^{\prime}\right|_{(x)}$ the number of cells after the effect of cycle $(x)$. This result illustrates the behavior of the cell population between cycles.

Rate of regrowth (2): Here, we calculated the ratio of the colony size at the end of each drug cycle, immediately preceding the next dosing, to its size at the beginning of the cycle, prior to drug application:

$$
\left.R_{2}\right|_{(x)}=\frac{\left.n\right|_{(x+1)}}{\left.n\right|_{(x)}} \times 100 .
$$

Time below threshold: In addition, we investigated the percentage of time in which the cell population was maintained below a certain threshold, determined as a proportion of its original size. For example, if we define the threshold $S_{1 / 2}$ as being half of the initial cell colony size, we compute the percentage of time the colony size is below $S_{1 / 2}$ as follows:

$$
P_{1 / 2}=\frac{\sum_{t=0}^{t=T} H\left(S_{1 / 2}-s(t)\right)}{T} \times 100
$$

where $P_{1 / 2}$ is the percentage of time when the colony is below half its initial size, $s(t)$ is the size of the population at time $t$ and $H$ is the Heaviside function:

$$
H(x)= \begin{cases}1 & \text { if } x>0 \\ 0 & \text { if } x<0 .\end{cases}
$$

The thresholds examined were $S_{3 / 4}, S_{1 / 2}$ and $S_{1 / 4}$. 


\section{Results}

\subsection{Blood flow heterogeneity}

Fig. 2 illustrates the effects of changing cellular patterns on nutrient flow. In the top figure, in which the domain is almost full of cells, the nutrient is distributed to some degree throughout the entire domain. In the bottom figure, in which we observe disaggregation of certain portions of the cell colony, the nutrient is concentrated almost completely in the area of the domain in which the cell pattern remains uniform. These effects demonstrate the heterogeneous blood flow caused by the vessel maturation/destabilization process [see Eq. (1)], which is a direct result of spatial rearrangement of cell population.

Blood vessel configuration and cellular pattern organization are mutually dependent. In densely populated areas the NHL colony incorporates and destabilizes nearby blood vessels. Because of this structural instability, blood flow in the vasculature becomes highly heterogeneous, causing irregular nutrient distribution. Rapid changes in nutrient distribution can cause large areas of the domain to become suddenly nutrient depleted, thus eliminating a significant proportion of cells and leading others into quiescence. When cellular patterns disaggregate the adjacent vessels become mature.

Heterogeneous blood flow causes significant oscillations in NHL cell population growth, as will be shown in the next section.

\subsection{Dynamics of an NHL cell colony without chemotherapy}

In order to fully appreciate the effect of Doxorubicin treatment on an NHL cell colony, it is necessary to understand its 'natural' behavior, i.e., when its dynamics are regulated only by nutrient flow. Fig. 3 illustrates the dynamics of HG NHL cells. The initial population in this case consists of 450 cells. This population increases by almost $30 \%$ within the first 50 time steps of the simulation, and then continues to oscillate around its increased value. This results from the fact that the colony cannot continue to grow indefinitely, due to limited nutrient availability and other constraints imposed by the domain's carrying capacity. ${ }^{4}$

The dynamics of the cell colony as depicted by Fig. 3 display a sharp decrease followed by gradually increasing plateau-like oscillations. As mentioned above, these significant phenomena illustrate the nature of the nutrient delivery, which results from the heterogeneous blood flow in the vasculature. A sharp decrease in population implies that an area has become suddenly nutrient depleted, causing a large number of cells to die. A increase followed by a plateau (see Fig. 3) indicates that an area that was previously nutrient depleted has begun to receive a sufficient nutrient supply. The fact that a large number of cells enter the cycle at the same time accounts for the synchronous growth of the population.

\subsection{Dynamics of an NHL cell colony under Doxorubicin treatment}

We simulated the effect of $10 \mathrm{mg} \mathrm{m}^{-2}$ Doxorubicin doses on NHL cell colonies of all three grades. In order to mimic actual chemotherapy protocols, simulations were carried

\footnotetext{
${ }^{4}$ Domain's maximum capacity: 3800 cells.
} 
Nutrient flow (mM/min)

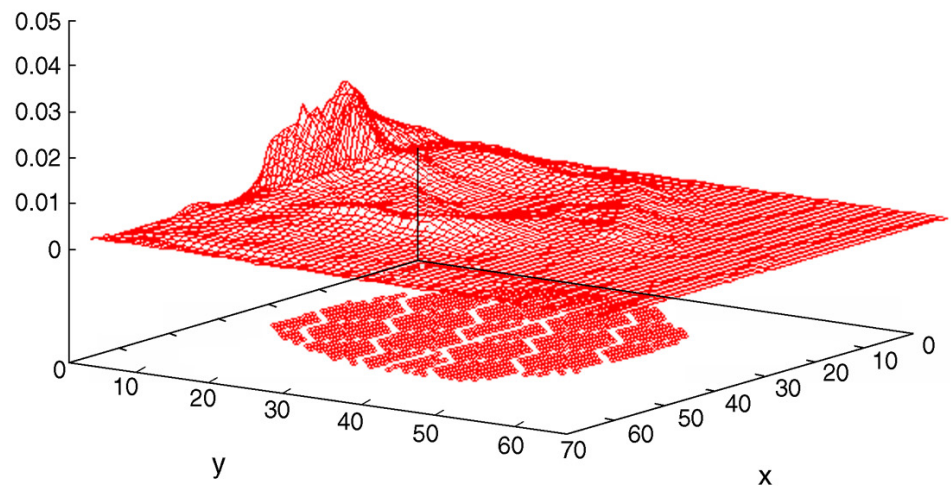

Nutrient flow $(\mathrm{mM} / \mathrm{min})$

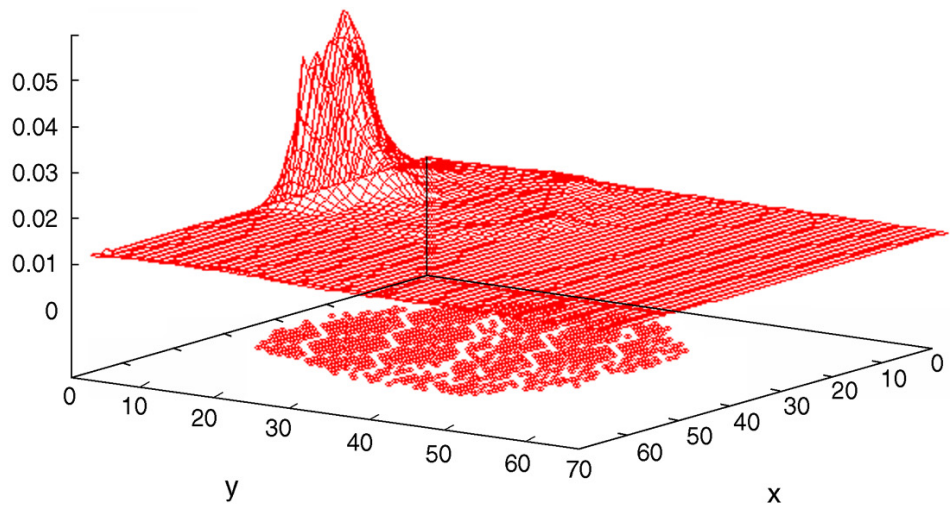

Fig. 2. Dynamics over time of nutrient flow and cell arrangement in a drug-free domain (figures separated by 65 h). 3D graphs: nutrient flow; 2D graphs: cell distribution (small circles). Flux is along the $y$ axis from right to left. Top: the cell arrangement is uniform; the vascular network is clearly visible (honeycomb structure). Bottom: following cell depletion in certain areas of the domain, the nutrient flow is significantly altered.

out over a period of $2600 \mathrm{~h}$ (approximately $3 \frac{1}{2}$ months), allowing six dose administrations separated by 21 days.

Due to the above-mentioned constraints of the model domain, the initial cell population was set randomly between 1000 and 1500 cells.

\subsubsection{Overall treatment effect}

The first aspect that we examined was the overall effect of the treatment on the NHL colony, i.e., the difference between the size of the population at the beginning and at the end of the simulation (see Table 4). LG NHL cells were not as affected by the treatment as the other grades: we observe a $48 \%$ decrease as opposed to $66 \%$ for IG and $62 \%$ for HG. 


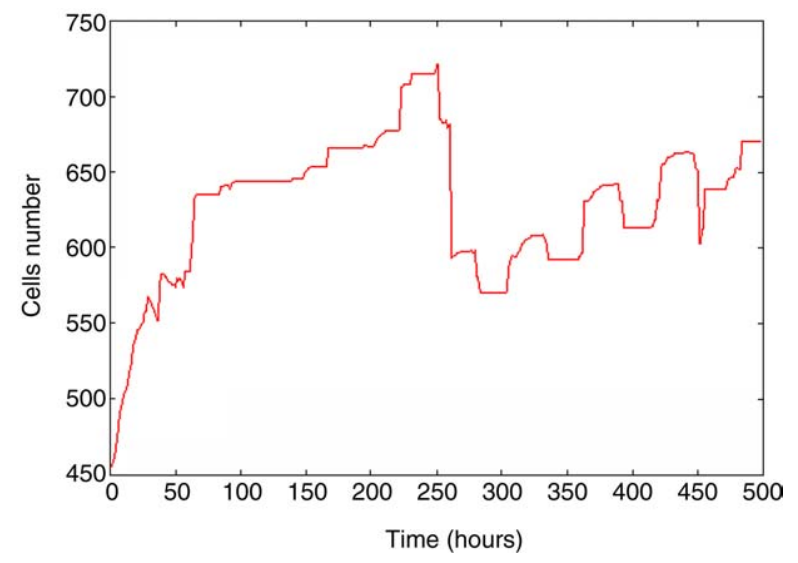

Fig. 3. Simulated HG NHL cell dynamics in a drug-free domain over $500 \mathrm{~h}$.

Table 4

The overall effect of Doxorubicin treatment on NHL cell colonies

\begin{tabular}{llll}
\hline & Initial & Final & Overall treatment effect $(\%)$ \\
\hline HG NHL & 1292 & 495 & -62 \\
IG NHL & 1398 & 474 & -66 \\
LG NHL & 1345 & 704 & -48 \\
\hline
\end{tabular}

The regimen consists of six cycles of $10 \mathrm{mg} \mathrm{m}^{-2}$ doses separated by 21 day intervals.

Table 5

Regrowth of NHL cell populations following periodic Doxorubicin applications (first method): $10 \mathrm{mg} \mathrm{m}^{-2} \mathrm{doses}^{-}$ separated by 21 day intervals

\begin{tabular}{llllll}
\hline Regrowth from: & 1st cycle $(\%)$ & 2nd cycle $(\%)$ & 3rd $(\%)$ & 4th $(\%)$ & 5th $(\%)$ \\
\hline HG NHL & 31 & 27 & 41 & 47 & 14 \\
IG NHL & 24 & 28 & 53 & 28 & 59 \\
LG NHL & 13 & 12 & 28 & 20 & 3 \\
\hline
\end{tabular}

\subsubsection{NHL cell regrowth after Doxorubicin application}

After each drug dose was administered, we computed the regrowth rate of the NHL cell population over the period of the drug cycle, i.e., the difference between the minimum number of cells following the injection (maximum drug effect) and the size of the population immediately prior to the next injection [see Eq. (12)]. All three grades of NHL displayed significant cell population recovery between drug cycles. The regrowth rate after each cycle for LG NHL was lowest at $15 \%$ on average, as opposed to $39 \%$ for IG NHL and $32 \%$ for HG NHL (Table 5). 
Table 6

Regrowth of NHL cell population following periodic Doxorubicin applications (second method): $10 \mathrm{mg} \mathrm{m}^{-2}$ doses separated by 21 day intervals

\begin{tabular}{llccc}
\hline Regrowth from: & 2nd cycle $(\%)$ & 3rd $(\%)$ & 4th $(\%)$ & 5th $(\%)$ \\
\hline HG NHL & 94 & 97 & 100 & 86 \\
IG NHL & 93 & 95 & 80 & 113 \\
LG NHL & 80 & 107 & 95 & 86 \\
\hline
\end{tabular}

In addition, we compared the size of the cell population at the beginning and at the end of each drug cycle. The figures displayed in Table $6^{5}$ represent the ratio of the population size at the end of the cycle, i.e., immediately preceding the next injection, to that at the beginning of the cycle, just before the previous drug application [see Eq. (13)]. For example, at the end of the fourth cycle, the HG NHL colony stood at $100 \%$ of its size just prior to the fourth drug application. According to Table 6, regardless of the grade of NHL, each drug application was followed by regrowth of at least $80 \%$. The average regrowth rate was $94 \%$ for HG, 95\% for IG and 92\% for LG. Regrowth of 100\% or more occurred for each grade, over different cycles: HG—after the fourth treatment; IG—after the fifth treatment; $\mathrm{LG}$-after the third treatment.

\subsubsection{Time below threshold}

Another aspect that we examined was the percentage of time in which the cell population remained beneath $S_{3 / 4}$ (3/4 initial size), $S_{1 / 2}$ and $S_{1 / 4}$ (see Table 7). During most of the treatment, the population was maintained within the range of $25 \%-75 \%$ of the original size. However, it did not decrease below this margin in any of the grades, and in the case of LG it did not decrease below 50\%. These results show a clear distinction between HG and IG: treatment succeeded in maintaining IG below $S_{1 / 2} 50 \%$ of the time, as opposed to $11 \%$ in the case of $\mathrm{HG}$.

\subsubsection{Growth patterns of cell populations}

Fig. 4 depicts the dynamics of HG, IG and LG NHL cell colonies under Doxorubicin treatment. If we examine the dynamics of the different cell populations between cycles, we observe behavior similar to that of the cell population without treatment: a rapid increase followed by gradually increasing oscillations. We note that the oscillations of the LG NHL population are less pronounced. In addition, the graphs illustrate clearly that the drug has less impact on LG NHL than on HG and IG as was implied by the previous results. These effects are due to the LG NHL colony's slow rate of growth. Moreover, the graphs demonstrate that IG NHL was more effectively repressed by the treatment than HG NHL: a significant difference in population recovery rate following the fourth cycle led the IG NHL colony size to remain below the $S_{1 / 2}$ threshold for the remainder of the simulation.

If we analyze the population regrowth after the Doxorubicin dosing, we can easily distinguish between two stages. The first is a period of rapid and constant growth, immediately following the drug effect. Then, approximately $200 \mathrm{~h}$ after the drug

\footnotetext{
5 Regrowth from the first treatment has not been displayed due to the random choice of the initial size.
} 
Table 7

The percentage of time for which the NHL cell population decreases below particular significant thresholds when Doxorubicin treatment is applied: six cycles of $10 \mathrm{mg} \mathrm{m}^{-2}$ doses separated by 21 day intervals

\begin{tabular}{llcc}
\hline & $S_{3 / 4}(\%)$ & $S_{1 / 2}(\%)$ & $S_{1 / 4}(\%)$ \\
\hline HG NHL & 94 & 11 & 0 \\
IG NHL & 99 & 50 & 0 \\
LG NHL & 80 & 0 & 0 \\
\hline
\end{tabular}
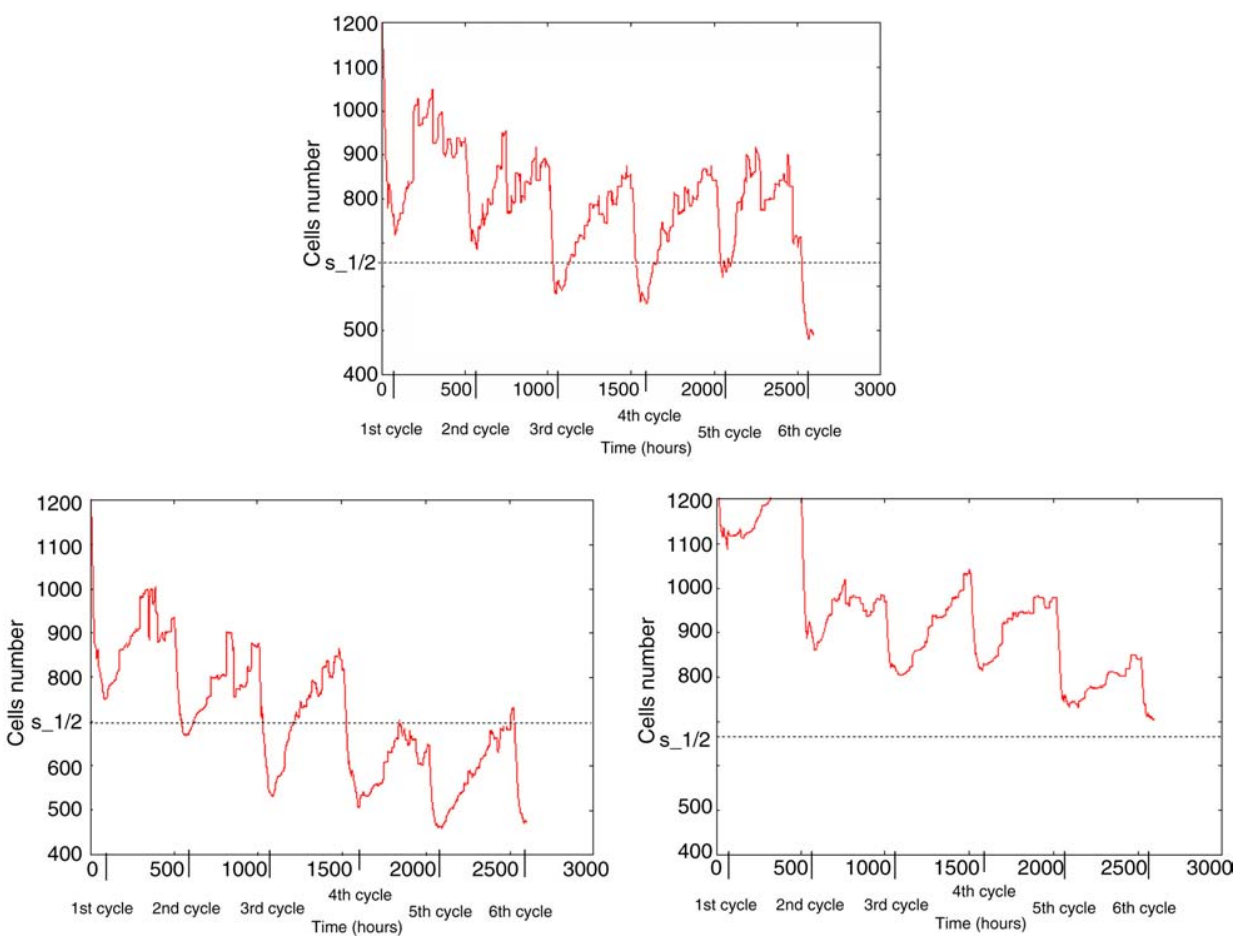

Fig. 4. From top to bottom right: HG NHL, IG NHL and LG NHL cell dynamics with Doxorubicin treatment: six cycles of $10 \mathrm{mg} \mathrm{m}^{-2}$ doses separated by 21 day intervals.

application, oscillations appear, often attaining considerable amplitudes. As mentioned above, oscillations are a reflection of blood flow heterogeneity. Due to the effects of the drug, cell clusters disaggregate, and the resulting decrease in overcrowdedness enables proliferation to accelerate (see CA rules). When new cell aggregations appear, they coopt vessels and modify their characteristics: engulfed vessels become immature and their radii are randomly changed [see Eq. (1)]. Consequently, blood flow becomes strongly irregular in the area. While the cell colony grows, a large proportion of vessels become immature, thus destabilizing the blood flow over the entire domain. Several areas of the domain become successively nutrient depleted, leading to cell death. The effects of this instability are displayed in Fig. 10, which compares NHL recovery from a chemotherapy cycle when 
vessel maturation/destabilization is implemented as opposed to when it is not. In the first case we observe significant oscillations in population size, whereas in the second the cell population increases at a steady pace.

\subsection{Model sensitivity to parameters}

It is not always possible to derive necessary parameter values directly from clinical data, especially in a model such as ours which is a two-dimensional representation of an actual NHL tumor cell colony. When estimating these values, we rely on additional data provided by the model that can be compared with clinical results (see for example Table 2). However, the potential influence of the choice of parameter values on the model's behavior cannot be disregarded.

The critical parameters that must be accounted for include:

- morphological parameters, i.e., blood vessel radii [ $r_{\text {mat }}$, Eq. (1)], which directly affect blood flow and distribution;

- the nutrient level below which cells go into quiescence ( $\left.n_{\text {block }}\right)$;

- the nutrient level below which cells die $\left(n_{\text {death }}\right)$.

First we examine the influence of these parameters on a 'global' level, by comparing the overall effect of the treatment on the cell colony. Fig. 5 presents the overall treatment effect obtained over $300 \mathrm{~h}$ following one drug application, when different values of these parameters are implemented. We can observe that the choice of $n_{\text {death }}$ has a significant effect on the simulation's outcome, whereas the ranges obtained by varying $n_{\text {block }}$ and $r_{\text {mat }}$ are more limited.

If we attempt to characterize the tumor's behavior under the influence of different parameter values, we discover additional effects.

We observe that increasing the value of $n_{\text {death }}$ causes the size of the NHL cell population at equilibrium to diminish. A population will only continue to grow as long as the level of available nutrients in its environment is able to sustain it. Therefore it is intuitively obvious that the higher the nutrient level required to maintain the population, the smaller that population will be. Fig. 6 demonstrates that when $n_{\text {death }}$ is increased to a certain point the population size continues to oscillate, though its point of equilibrium is lower. However, if $n_{\text {death }}$ is increased beyond this threshold, the cell colony's point of equilibrium is so low that it is virtually unable to grow after the initial drug application, and its size remains almost constant. The lack of growth indicates that there are few or no changing cell patterns, thus reducing blood flow heterogeneity and eliminating oscillations.

If we modify $r_{\text {mat }}$ and $n_{\text {block }}$, we observe that regardless of the value implemented, the development of the colony can still be clearly divided into two stages as described earlier, i.e., a period of constant growth followed by oscillations (see Figs. 7 and 8). However, different parameter values may affect the amplitude or other specific qualities of the oscillations.

We emphasize that all estimated parameter values implemented in the model were derived in accordance with clinical data, e.g., expected growth fraction (Brons et al., 1992). 


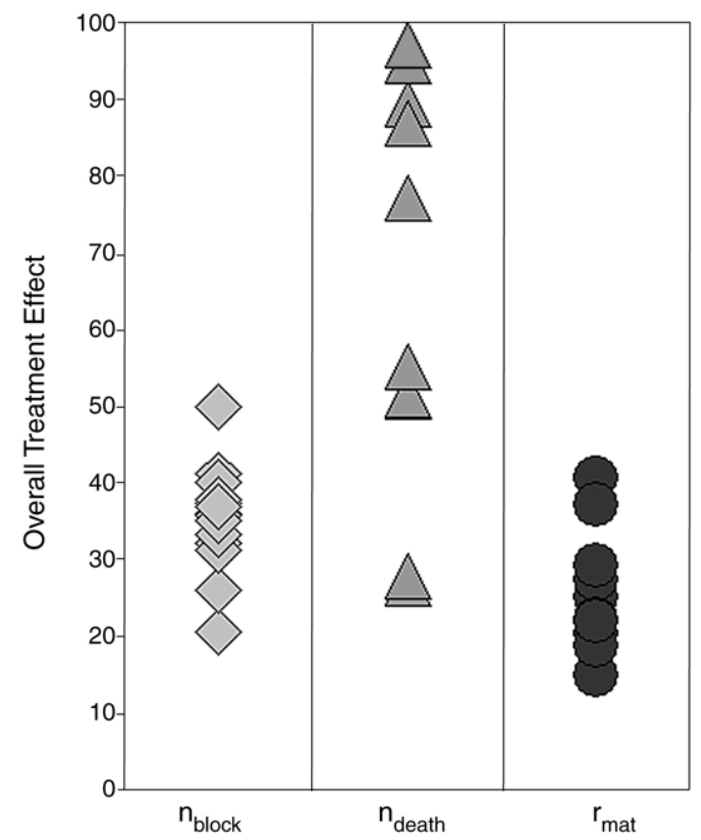

Fig. 5. A histogram illustrating the overall treatment effect on HG NHL, $300 \mathrm{~h}$ after Doxorubicin administration. Each symbol (diamonds, triangles and circles) represents the overall treatment effect obtained when its respective parameter value $\left(n_{\text {block }}, n_{\text {death }}, r_{\text {mat }}\right)$ was modified within a feasible range.

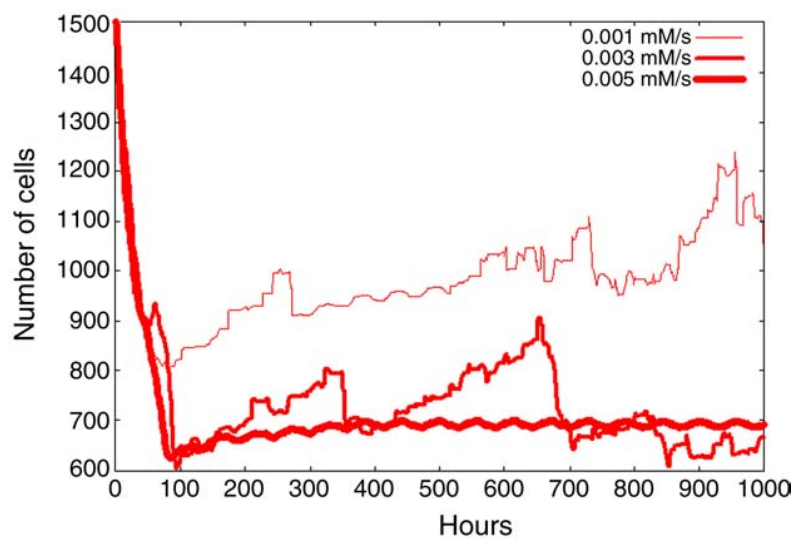

Fig. 6. Simulated HG NHL dynamics over $1000 \mathrm{~h}$ with one drug application at $t=0 . n_{\text {death }}$ taken at $0.001 \mathrm{mM} \mathrm{s}^{-1}$ (the value implemented in simulations), at $0.003 \mathrm{mM} \mathrm{s}^{-1}$ and at $0.005 \mathrm{mM} \mathrm{s}^{-1}$.

\section{Discussion}

We presented a modelling framework and simulations aimed at predicting the qualitative effect of Doxorubicin administration on NHL cell colonies. Our model takes into account 


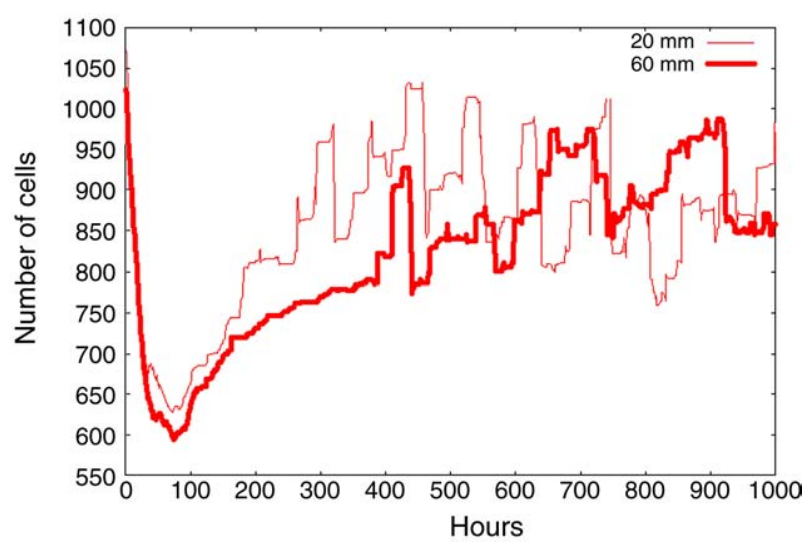

Fig. 7. Simulated HG NHL dynamics over $1000 \mathrm{~h}$ with one drug application at $t=0 . r_{\text {mat }}$ taken at $20 \mu \mathrm{M}$ (the value implemented in simulations) and at $60 \mu \mathrm{M}$.

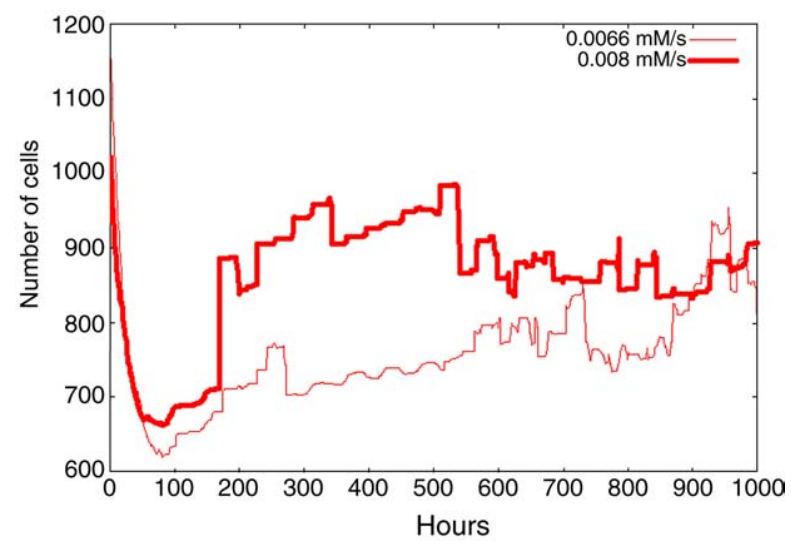

Fig. 8. Simulated HG NHL dynamics over $1000 \mathrm{~h}$ with one drug application at $t=0 . n_{\text {block }}$ taken at $0.0066 \mathrm{mM} \mathrm{s}^{-1}$ (the value implemented in simulations) and at $0.008 \mathrm{mM} \mathrm{s}^{-1}$.

principal biological features such as NHL cell-cycle kinetics, Doxorubicin PK/PD and blood flow heterogeneity.

The simulation results which most accurately reflect treatment efficacy are the population regrowth rate between cycles and the percentage of time for which the population is maintained below a certain threshold. The overall treatment effect, while informative when used to compare between the different grades, does not provide a true indication of efficacy. This is due to the fact that measurement is begun on a random colony which has not necessarily reached equilibrium, and is stopped at an arbitrary time step.

Comparison between simulations of the different grades of NHL shows treatment to be least effective in the case of LG NHL, which displayed a high cell regrowth rate in addition to a relatively weak drug impact (Table 5). This behavior is due to the low growth fraction 


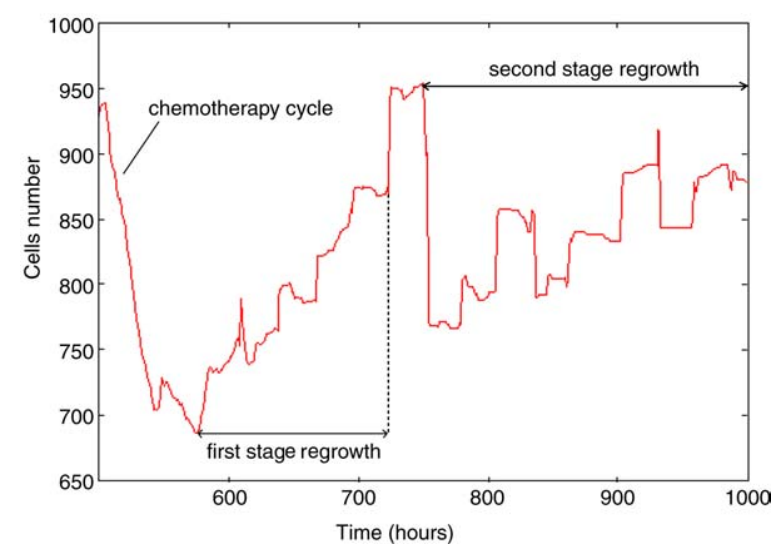

Fig. 9. An illustration of the two distinct stages of colony regrowth following a $10 \mathrm{mg} \mathrm{m}^{-2}$ Doxorubicin administration.

of LG NHL cells. This result has been reflected in clinical studies: LG NHL patients are treated using a different protocol (Al-Ismail et al., 1987).

Furthermore, simulations indicate that treatment was inefficient on all three grades of NHL. HG, IG and LG NHL displayed significant population regrowth following the effect of each drug application. In some cases, by the end of the drug cycle the cell colony managed to recover to a population exceeding that prior to the drug administration.

Blood flow heterogeneity appeared to be a key factor in these results. Simulations show that a colony's regrowth following the application of Doxorubicin can be divided into two stages: a rapid and regular growth (first-stage regrowth) until aggregation of cells leads to a large proportion of vessels becoming immature and thus structurally unstable [see Eq. (1)]. This structural instability leads to blood flow becoming extremely perturbed, causing consecutive regions to become nutrient depleted. The result is a pattern of significant oscillations in population size (second stage regrowth) (see Fig. 9).

The effect of blood flow heterogeneity is illustrated in Fig. 10, which compares cell recovery from a chemotherapy cycle when the vessel maturation/destabilization process is taken into account, compared to a case in which this assumption is relaxed.

Applying an additional dosing while the system is oscillating will not improve the effect, though a significant impact may occur occasionally if the new cycle is administered while the system is at the minimum of the oscillation (see Fig. 9). However, efficacy can be systematically improved if the new cycle of Doxorubicin treatment is applied while recovery is still at its first stage. Fig. 11 illustrates the model prediction following such an application protocol on HG NHL.

We are thus led to the conclusion that in order to optimize the effect of Doxorubicin treatment, it is not enough to merely reduce the dosing interval. The interval must be reduced to a point at which additional drug cycles are applied before the NHL cell colony has a chance to enter the unstable, oscillating stage of its recovery (see Fig. 11). Note however that the current work does not consider possible toxic effects of the drug. 


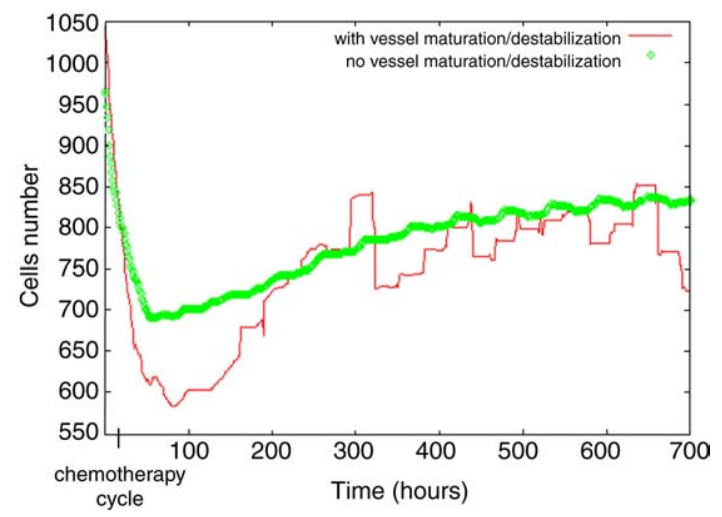

Fig. 10. The effect of the vessel maturation/destabilization process on the cell population recovery following a $10 \mathrm{mg} \mathrm{m}^{-2}$ Doxorubicin administration. Thin line: with vessel maturation/destabilization; empty circles (thick line): no vessel maturation/destabilization is assumed.

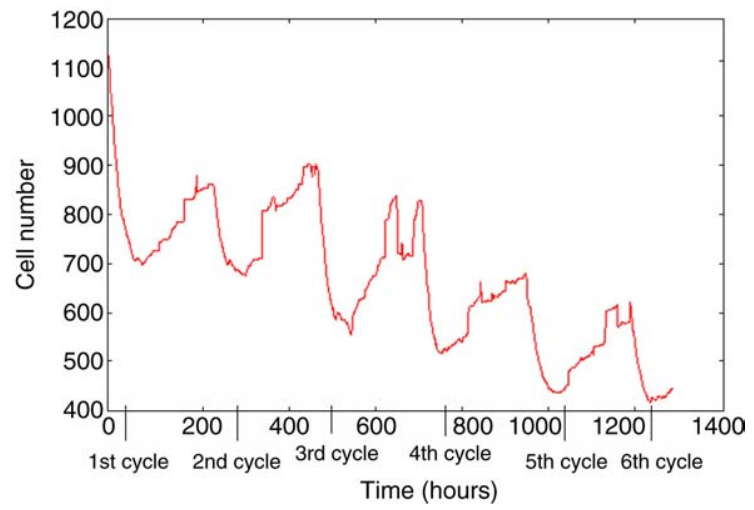

Fig. 11. HG NHL cell dynamics following Doxorubicin densified treatment: six cycles of $10 \mathrm{mg} \mathrm{m}^{-2}$ doses separated by 10 day intervals.

Can biological vascular instability generate significant changes in blood supply in a short time (less than one hundred hours) as depicted in Fig. 2?

We are not aware of any clinical studies estimating the timescale upon which the effects of blood flow heterogeneity occur. However, the existence of a transition point prior to which treatment needs to be applied in order to achieve the optimal effect seems an intuitive conclusion. Figs. 6-8 indicate that this conclusion is robust: regardless of specific parameter values implemented, this transition point can be observed as long as the NHL cell colony continues to grow.

Clearly our results are qualitative and should be interpreted as such. In order to provide any quantitative indication of the effect of Doxorubicin on non-Hodgkin's lymphoma, a three-dimensional structure and a larger domain enabling simulation of actual standard 
drug doses must be considered. However, the study does illustrate the significant influence of blood flow heterogeneity on the effect of Doxorubicin treatment.

\section{Acknowledgements}

The work was supported by the EU Research Training Network (5th FrameworkHPRN-CT-2000-00105): 'Using mathematical modelling and computer simulation to improve cancer therapy' and by the Chai Foundation. We wish to acknowledge particularly Nitsan Dahan, Dr. Vladimir Vainstain, Yuri Kogan and Vera Sleitzer for useful discussion, Dr. Helen Byrne ${ }^{6}$ for her involvement in the initial stages of the modeling and Dr. Filippo Castiglione for valuable advice regarding the model implementation and exploitation. We would also like to thank the referees for their useful comments.

\section{References}

Agur, Z., 1985. Randomness, synchrony and population persistence. J. Theor. Biol. 112, 677-693.

Agur, Z., Arnon, R., Schechter, B., 1988. Reduction of cytotoxicity to normal tissues by new regimens of phasespecific drugs. Math. Biosci. 92, 1-15.

Alarcón, T., Byrne, H.M., Maini, P.K., 2003. A cellular automaton model for tumor growth in inhomogeneous environment. J. Theor. Biol. 225, 257-274.

Alarcón, T., Byrne, H.M., Maini, P.K., Design principle of vascular beds: effects of blood rheology (in preparation).

Alberts, B., Bray, D., Lewis, J., Raff, M., Roberts, K., Watson, J.D., 1994. Molecular Biology of the Cell. Garland Publishing, New York and London.

Al-Ismail, S.A., Whittaker, J.A., Gough, J., 1987. Combination chemotherapy including Epirubicin for the management of non-Hodgkin's lymphoma. Eur. J. Cancer Clin. Oncol. 23, 1379-1384.

Anderson, A.R.A., Chaplain, M.A.J., 1998. Continuous and discrete mathematical models of tumour-induced angiogenesis. Bull. Math. Biol. 60, 857-899.

Anderson, A.R.A., Chaplain, M.A.J., Newman, E.L., Steele, R.J.C., Thompson, A.M., 2000. Mathematical modelling of tumor invasion and metastasis. J. Theor. Med. 2, 129-154.

Arakelyan, L., Vainstein, V., Agur, Z., 2002. A computer algorithm describing the process of vessel formation and maturation and its use for predicting the effects of anti-angiogenic and multi-maturation therapy on vascular tumor growth. Angiogenesis 5, 203-214.

Barranco, S.C., 1984. Cellular and molecular effects of Adriamycin on dividing and nondividing cells. Pharmacol. Ther. 24, 303-319.

Bast, R.C., Kufe, D.W., Pollock, R.E., Weichselbaum, R.R., Holland, J.F., 2000. Cancer Medicine. BC Decker Inc, Canada.

Benjamin, L.E., Golijanin, D., Itin, A., Pode, D., Keshet, E., 1999. Selective ablation of immature blood vessels in established human tumors follows vascular endothelial growth factor withdrawal. J. Clin. Invest. 103, $157-158$.

Blagosklonny, M.V., Pardee, A.B., 2002. The restriction point of the cell cycle. Cell Cycle 1, 103-110.

Boring, C.C., Squires, T.S., Tong, T., Montgomery, S., 1994. Cancer statistics, 1994. C.A. Cancer J. Clin. 44, 7-26.

Brons, P.P., Raemaekers, J.M., Bogman, M.J., van Erp, P.E., Boezeman, J.B., Pennings, A.H., Wessels, H.M, Haanen, C., 1992. Cell cycle kinetics in malignant lymphoma studied with in vivo Iodeoxyuridine administration, nuclear Ki-67 staining, and flow cytometry. Blood 1 80, 2336-2343.

\footnotetext{
${ }^{6}$ H.M. Byrne, Centre for Mathematical Medicine, School of Mathematical Sciences, University of Nottingham, Nottingham NG7 2RD, UK.
} 
Casciari, J.J., Sotirchos, S.V., Sutherland, R.M., 1998. Glucose diffusivity in multicellular tumor spheroids. Cancer Res. 48, 3905-3909.

Cojocaru, L., Agur, Z., 1992. A theoretical analysis of interval drug dosing for cell-cycle-phase-specific drugs. Math. Biosci. 109, 85-97.

Couderc, B., Dujols, J.P., Mokhtari, F., Norkowski, J.L., Slawinski, J.C., Schlaifer, D., 2000. The management of adult aggressive non-Hodgkin's lymphomas. Crit. Rev. Oncol. Hematol. 35, 33-48.

Crone, C., Levitt, D.G., 1984. Handbook of Physiology: A Critical, Comprehensive Presentation of Physiological Knowledge and Concepts. American Physiological Society, Bethesda, ML.

Deutsch, A., Dormann, S., 2002. Modeling of avascular tumor growth with a hybrid cellular automaton. In Silico Biol. 2, 1-14.

Enden, G, Popel, A.S., 1994. A numerical study of plasma skimming in small vascular bifurcations. J. Biomech. Eng. 116, 79-88.

Erlanson, M., Lindth, J., Zackrisson, B., Landberg, G., Roos, G., 1995. Cell kinetic analysis of non-Hodgkin's lymphomas using in vivo Iodeoxyuridine incorporation and flow cytometry. Hematol. Oncol. 13, 207-217.

Hackbusch, W., 1985. Multigrid Methods and Applications. Springer, Berlin.

Hardman, J.G., Limbird, L.E., Gilman, A.G., 2001. The Pharmacological Basis of Therapeutics. McGraw-Hill Companies.

Honda, H., Yoshizato, K., 1997. Formation of branching pattern of blood vessels in the wall of the avian yolk sac studied by computer simulation. Dev. Growth Differ. 39, 581-589.

Jain, R.K., 2001. Delivery of molecular and cellular medicine to solid tumours. Adv. Drug. Deliv. Rev. 46, 146-168.

Konerding, M.A., Fait, E., Gaumann, A., 2001. 3D microvascular architecture of pre-cancerous lesions and invasive carcinomas of the colon. Br. J. Cancer. 84, 1354-1362.

Kwok, T.T., Twentyman, P.R., 1985. The response to cytotoxic drugs of EMT6 cells treated either as intact or disaggregated spheroid. Br. J. Cancer. 51, 211-218.

Lankelma, J., Luque, R.F., Dekker, H., Schinkel, W., Pinedo, H., 2000. A mathematical model of drug transport in human breast cancer. Microvasc. Res. 59, 149-161.

Lapela, M., Leskinen, S., Minn, H.R., Lindholm, P., Klemi, P.J., Soderstrom, K.O., Bergman, J., Haaparanta, M., Ruotsalainen, U., Solin, O., 1995. Increased glucose metabolism in untreated non-Hodgkin's lymphoma: a study with positron emission tomography and fluorine-19-fluorodeoxyglucose. Blood 1 86, 3522-3527.

Lepage, E., Gisselbrecht, C., Haioun, C., Sebban, C., Tilly, H., Bosly, A., Morel, P., Herbrecht, R., Reyes, F., Coiffier, B., 1993. Prognostic significance of received relative dose intensity in non-Hodgkin's lymphoma patients: application to LNH-87 protocol. The GELA. (Groupe d'Etude des Lymphomes de l'Adulte). Ann. Oncol. 4, 651-656.

Liotta, L.A., Saidel, G.M., Kleinerman, J., 1977. Diffusion model of tumor vascularization and growth. Bull. Math. Biol. 39, 117-128.

McCormick, S.F., 1987. Multigrid Methods. SIAM, Philadelphia.

McDougall, S.R., Anderson, A.R.A., Chaplain, M.A.J., Sherratt, J.A., 2002. Mathematical modelling of flow through vascular networks: Implications for tumour-induced angiogenesis and chemotherapy strategies. Bull. Math. Biol. 64, 673-702.

Nagai, K., Nagasawa, K., Sadzuka, Y., Tsujimoto, M., Takara, K., Ohnishi, N., Yokoyama, T., Fujimoto, S., 2002. Relationships between the in vitro cytotoxicity and transport characteristics of Pirarubicin and Doxorubicin in M5076 ovarian sarcoma cells, and comparison with those in Ehrlich ascites carcinoma cells. Cancer Chemother. Pharmacol. 49, 244-250.

Orme, M.E., Chaplain, M.A.J., 1996. A mathematical model of vascular tumour growth and invasion. Math. Comput. Modelling 23, 43-60.

Patel, A.A., Gawlinski, E.T., Lemieux, S.K., Gatenby, R.A., 2001. A cellular automaton model of early tumor growth and invasion. J. Theor. Biol. 7 213, 315-331.

Pries, A.R., Secomb, T.W., Gaehtgens, P., 1998. Structural adaptation and stability of microvascular networks: theory and simulations. Am. J. Physiol. 275, H349-H360.

Pries, A.R., Secomb, T.W., Gessner, T., Sperandio, M.B., Gross, J.F., Gaehtgens, P., 1994. Resistance to blood flow in microvessels in vivo. Circ. Res. 75, 904-915.

Stokke, T., Holte, H., Smedshammer, L., Smeland, E.B., Kaalhus, O., Steen, H.B., 1998. Proliferation and apoptosis in malignant and normal cells in B-cell non-Hodgkin's lymphomas. Br. J. Cancer 77, 1832-1838. 
Willemse, F., Nap, M., de Bruijn. H.W.A., Hollema, H., Morphological parameters of vasculature in tumor marker biodynamics. Anal. Quant. Histol. (in press).

Wilson, J.D, Foster, D.W., 1992. Williams Textbook of Endocrinology. W.B. Saunders Company.

Yancopoulos, G.D., Davis, S., Gale, N.W., Rudge, J.S., Wiegand, S.J., Holash, J., 2000. Vascular specific growth factors and blood vessel formation. Nature 407, 242-248. 